\title{
Н.А.КОСОЛАПОВ \\ ОБЗОР АКТУАЛЬНЫХ \\ ИССЛЕДОВАНИЙ ЗВУКА В ГОРОДЕ
}

Urban Studies and Practices Vol.2 \#4, 2017, 59-68

https://doi.org/10.17323/usp24201759-68

Косолапов Никита Алексеевич, магистрант Высшей школы урбанистики имени А.А. Высоковского НИУ ВШЭ; Российская Федерация, 101000, Москва, ул. Мясницкая, д. 13, стр. 4.

E-mail: nakosolapov@gmail.com

Статья посвящена описанию научных работ, выполненных в области исследований звука в городе в 20152018 гг. Отправной точкой для анализа послужил архив междисциплинарного журнала “Sound Studies”, однако помимо этого в обзоре подробно описаны научные статьи, опубликованные в других журналах за тот же период. Особое внимание уделяется постановке исследовательских вопросов в работах, подходам к их решению и сравнению полученных результатов.

Ключевые слова: исследования звука; исследования города; междисциплинарные исследования; научные журналы

$\mathrm{K}$ лассические определения города, как правило, отталкиваются от противопоставления города и деревни (см., например, [Вебер, 2001; Вирт, 2005] или [Mumford, 1938]). При этом базовые признаки города всегда остаются более или менее общими: границы, численность и плотность населения, сложные экономические связи. С 1930 - 1950-х годов, когда эти определения были даны, города обросли некоторым количеством других присущих им черт. Одной из них является звук города. Шум автомобилей, игра уличных музыкантов, звонки мобильных телефонов, аудиореклама и музыка в общественных пространствах - все это является неотъемлемой частью городской жизни, а порой - навязчивым ее атрибутом. В течение длительного времени звук в городе оставался объектом исследования лишь как физическое явление, в контексте безопасности или экологии (см., например, [Bullen, Fricke, 1976]). Только с середины 1970-х годов, после выхода в свет работы канадского музыканта и педагога Рэймонда Мюррея Шейфера “Тhe Soundscape: Our Sonic Environment and the Tuning of the World" [Schafer, 1977] звук как таковой и звук в городе в частности стал рассматриваться как явление социальное.
Институциализация sound studies - исследовательской области, посвященной изучению звука во всей его сложности (как физического, социального, музыкального, психологического явления), связана с появлением междисциплинарного журнала “Sound Studies”, первый номер которого вышел в 2015 г. В описании журнала на сайте издательства Taylor \& Francis ${ }^{1}$ сказано, что издание «поощряет исследования в области звука, публикуя междисциплинарные, теоретические, эмпирически обоснованные и критические работы». Значительную часть архива журнала составляют статьи, посвященные звуку в контексте города и архитектуры. Кажется важным сделать подробный обзор этих работ и попытаться понять, каким образом изучение звука в городской среде обогащает наше знание о городе и его жителях. Какой потенциал для развития urban studies и практик городского планирования и управления содержат в себе исследования звука. При этом наша картина едва ли будет полной без описания исследований по теме, опубликованных за время существования журнала “Sound Studies” в других изданиях.

1 Taylor \& Francis Online. Sound Studies: An Interdisciplinary Journal. CM.: https://www. tandfonline.com/loi/rfso20. 
Приступая к работе, мы определили алгоритм выбора статей. В первую очередь в перечень анализируемых научных работ вошли статьи из архива журнала “Sound Studies”, так или иначе связанные с городскими исследованиями. В ходе работы стало очевидно, что развитие области исследований звука в городе отнюдь не ограничено этими статьями. Отбор исследований, опубликованных в других изданиях, был ограничен двумя критериями. Во-первых, научные журналы, в которых выпущены эти статьи, находятся в первом или втором квартиле рейтинга Scimago по urban studies (среди них - журналы Cities, Journal of Urban and Regional Research, Landscape and Urban Planning и др.). Во-вторых, работы относятся к периоду издания журнала "Sound Studies" (и, как следствие, институционализированного существования этой исследовательской области) - 2015-2018 гг. Необходимо сказать, что эти ограничения были достаточно условными, потому как некоторые статьи, кажущиеся нам важными и необходимыми для этого обзора, опубликованы в журналах по политической науке или cultural studies. Работа структурирована следующим образом. В первой части мы уделим внимание исследованиям городского звука, опубликованным в журнале "Sound Studies". Во второй части текста будет показано, как развивалась эта исследовательская область в публикациях других журналов.

Журнал “Sound Studies" - An Interdisciplinary Journal выпускается издательством Taylor \& Francis дважды в год с 2015 г. К настоящему времени вышли в свет пять номеров. Журнал позиционирует себя как площадку для развития исследований в области звука и публикует работы на стыке нескольких дисциплин. Среди 52 статей из архива журнала работы о звуке в городе занимают примерно пятую часть, и их описанию мы хотели бы посвятить первую часть нашей работы.

Первая из них - "Landscapes and gimmicks from the 'sounded city': listening for the nation at the sound archive" [Madrid, 2016] - является не столько статьей, сколько эссе. Автор рассматривает опыт создания фонотеки Института национальной антропологии и истории в Мехико в 1964 г. Привлекательная черта этого проекта состоит в стремлении не только сохранить и систематизировать аудиозаписи звуковых фрагментов, отражающих национальную идентичность мексиканцев, но и применить их в образовательных и куль- турных инициативах. С темой города связан один из проектов фонотеки - звуковая карта Мехико, создание которой было инициировано в 2010 г. как попытка обратить внимание аудитории на повседневный городской звуковой фон. К концу 2015 г. участники проекта - горожане и сотрудники фонотеки - загрузили на интерактивную карту более 380 звуковых файлов совершенно разного происхождения: это были звуки природы, шумы местных традиционных праздников, рынков. Автор эссе рассматривает этот проект и фонотеку в целом в контексте идеи о звуке как эпистеме [Foucalt, 1970]. Развивая понятие эпистемы, концепция “lettered city” утверждает, что литературная деятельность и владение печатным словом вообще являются отличительным признаком привилегированной социальной группы, создающей культурный капитал. Звук же, в понимании автора, представляет собой более непосредственный способ создания этого культурного капитала, потому как он универсален и не требует знания определенного кода как для создания, так и для освоения созданного. В этом смысле создание подобных архивов-фонотек - необходимая практика, позволяющая решить проблему сохранения и воспроизводства национальной идентичности, например, через образовательные и культурные проекты.

Работы по истории повседневности занимают значительное место среди исследований в социальных науках в целом, и наиболее надежным источником для автора здесь становятся документы, относящиеся к изучаемой эпохе. Когда дело касается изучения звука, такими документами в первую очередь являются архивные аудиофрагменты. Проект The Roaring Twenties - это и есть такой архив, своего рода медийное приложение и продолжение книги “The Soundscape of Modernity: Architectural Acoustics and the Culture of Listening in America, 1900-1933" американского историка Эмили Томпсон [Thompson, 2004]. Морат [Morat, 2016] в своей работе описывает это собрание аудиозаписей звукового ландшафта Нью-Йорка первой трети XX в. как уникальный результат кропотливой работы: каждый отрывок сопровождается кратким описанием и комментарием. Пользователю доступна навигация по типу шума, времени и месту записи. Мысль о том, что разнообразный шум явился неотъемлемым атрибутом урбанизации, кажется очевидной, однако частью научного дискурса она может стать 
лишь в том случае, если у нее есть некоторое материальное подтверждение. Подобного рода звуковой архив играет в том числе и такую роль. Более того, опубликованные аудиозаписи зафиксировали и реакцию горожан на какофонию городской жизни, и неоднозначность восприятия горожанами явлений, которые возникли вследствие роста городов, таких как строительство небоскребов, развитие общественного транспорта, по сути, показывает неоднозначность самого процесса урбанизации.

Диджитальные академические исследования звука представлены проектом “Provoke! Digital Sound Studies”, описанным в статье Герлофа [Gerloff, 2016]. Аспиранты Duke University продолжили традицию архивирования звуков, используя цифровые технологии. Подобрав совершенно разные по своей природе материалы - оборудование для усиления и обработки звучания музыкальных инструментов, звуковые и материальные артефакты, собранные на острове Гаити в 2010 г., за три дня до землетрясения, исследователи задались вопросом, что можно узнать о звуке, используя исключительно звук. Автор обзорной статьи утверждает, что этот и подобные проекты в целом важны по двум причинам. Во-первых, такой формат расширяет возможности для презентации академических исследований. Больше того - эти инновации позволяют исследователю стереть границы между привычной формой академического исследования, научно-популярной литературой и журналистской работой - просто потому, что выполненный в определенном стиле письменный текст здесь не является ключевым элементом в изложении результатов проведенной работы.

Статья Гэллуэя [Galloway, 2016] описывает перфоманс “The Harbour Symphony”, ежегодно проводимый на острове Ньюфаундленд с 1983 г. Замысел этого экспериментального музыкального произведения заключается в том, что основными «инструментами» в нем становятся гудки стоящих в порту кораблей и другие естественные для этого места звуки. По словам создателей, этот перформанс призван объединить город, природу и общество, а также подчеркнуть уникальную физическую и акустическую среду, созданную в гавани. Авторами музыкальных произведений, исполняемых в ходе действа, являются профессиональные композиторы. Слушателями симфонии становятся все жители горо- да. Проведя серию интервью со слушателями симфонии, автор делает следующее заключение: опыт слушателей зависел от того, в какой части города они слушали симфонию; респонденты отмечали, что изменяющаяся окружающая среда, как физическая, так и звуковая (например, шум проезжающих машин), влияли на восприятие ими произведения.

Некоторые статьи из архива журнала Sound Studies, посвященные звуку в городе, не содержат эмпирического исследования как такового. На наш взгляд, такие работы требуют меньшего внимания, однако не могут быть исключены из обзора, претендующего на некоторую полноту и обстоятельность. В эту категорию можно отнести эссе Ньергеса [Nyerges, 2016], посвященное анализу поэмы “Voyages” американского поэта Харта Крейна и тому, какое место городской звук НьюЙорка занимает в ней. Шум Нью-Йорка 1920-х годов был частью того культурного ландшафта, который поэты-модернисты пытались осмыслить и запечатлеть в своей поэзии. Автор пытается показать на этом примере, как в поэзии данного направления отражены пространство и звук: они тесно переплетены друг с другом, более того - время от времени звук как бы обретает форму, становясь частью физического ландшафта.

К этой же категории относятся рецензии на события, так или иначе связанные со звуком в городе, - работа Катрин Дрекманн [Dreckmann, 2017] описывает выставку, посвященную музыкальным традициям Рейнской области, а статья Переведенцевой и Гарсиа [Perevedentseva, Garcia, 2016] - экспозицию коллекции виниловых пластинок чикагского диджея Фрэнки Наклза.

Начиная работу над этим обзором, мы полагали необходимым ограничиться описанием статей из архива журнала “Sound Studies”. Однако уже на этапе беглого ознакомления с содержанием архива стало понятно, что работы чаще всего лишены значимой эмпирической составляющей, и по крайней мере в части изучения звука в городе журнал не выполняет задачи институционализации исследовательской области. Изученная нами часть портфолио издания может быть охарактеризована как популярная наука, эссеистика, но не как строгое научное знание о звуке в городе. Именно в связи с этим было принято решение рассмотреть ведущие издания в области городских исследований и смежных дисциплин, чтобы составить более полную 
и объективную картину того, как развивались исследования звука в городе в последние годы. Далее мы разберем последовательно кейсы, эмпирические качественные и количественные исследования, а затем отдельно работы, относящиеся к тематике звука в городском планировании.

В 2004 г. на публичных слушаниях в католическом городке Хамтрак, штат Мичиган, обсуждался вопрос о возможности звучания на улицах азана - публичного призыва мусульман к молитве. Эта инициатива вызвала противоречивую реакцию городского сообщества: для одной части населения она показалась удачным инструментом интеграции меньшинства, в то время как для другой символизировала захват города мусульманами (после 9/11 прошло всего три года). В результате общественного обсуждения мусульманские призывы к молитве все же зазвучали на улицах города. Одновременно власти предложили проводить открытые религиозные мероприятия, чтобы побудить людей различной религиозной принадлежности включаться в прежде эксклюзивные духовные практики отдельных конфессий и таким образом расширять свои культурные границы. Статья Перкинс [Perkins, 2015] подробно описывает ход публичной дискуссии по этому вопросу и аргументацию сторон. Помимо того что этот кейс являет собой случай цивилизованной процедуры общественного выбора, и уже одно это делает его ценным, он позволяет через феномен звука в городе по-новому посмотреть на привычные для городских исследований понятия. Вроде бы кажется очевидным, что пресловутое «право на город» касается преобразования не только физического пространства, но и других составляющих публичных пространств. Однако чаще этот термин возникает именно в контексте обсуждений градостроительных инициатив. Кроме того, случай иллюстрирует менее радикальное, подразумевающее применение так называемой мягкой силы, но попрежнему продуктивное воплощение права на город, по сравнению с тем, которое предлагает один из авторов термина Дэвид Харви [Harvey, 2003].

Столкновение интересов разных групп городского населения в контексте звука обсуждает и работа “If I Was King Of India I Would Get All The Horns Out Of Cars": A Qualitative Study of Sound In Delhi [Patsarika, Schneider, Edwards, 2018]. Процесс урбанизации в стра- нах глобального Юга привлекает гораздо меньший интерес академического сообщества, чем вопросы развития европейских или американских городов. Между тем африканские, азиатские и латиноамериканские страны сталкиваются с проблемами, аналогичными европейским: перенаселение, социальная дифференциация, загрязнение воздуха, воды и, в частности, звуковой среды городов. В статье Патсарики, Шнайдер и Эдвардса речь идет об индийском городе Дели. Знаковая составляющая его звукового ландшафта - сигнал автомобильного гудка. С одной стороны, социальные и культурные нормы культивируют лояльное отношение к громкому звуку, с другой - будучи воспроизводимым тысячами машин, звук подвергает опасности здоровье тех, кто живет поблизости от крупных магистралей и работает на улице (продавцы, перевозчики и проч.). Авторы статьи описывают подходы к решению проблемы, к которым прибегало городское сообщество. Один из них - кампания “Do Not Honk”, направленная на использование менее шумных транспортных средств и развитие культуры сравнительно тихого пользования автомобилем. Неудачу инициативы авторы объясняют тем, что в ее реализацию были вовлечены только новый средний класс и состоятельное население, остальные жители не имели возможностей поучаствовать в кампании. В исследовании приняли участие 75 информантов: они прослушали ряд аудиофрагментов из повседневного звукового контекста в разных частях города, а затем в рамках глубинного интервью описали свое впечатление от услышанного. Отвечая на вопрос о пользовании автомобильным сигналом, они говорили о том, что, с одной стороны, это воплощение власти и богатства: только состоятельный человек может позволить себе владеть машиной; с другой стороны, гудок автомобиля привычен как для тех, кто его производит, так и для тех, кто его слышит. Описанный кейс позволяет сделать два общих вывода. Во-первых, для горожан звук может быть маркером социально-экономического статуса. Во-вторых, в отдельных случаях звук становится частью идентичности горожанина и образа города.

Работа Мерилл “The Life of a Gunshot: Space, Sound and the Political Contours of Acoustic Gunshot Detection” [Merrill, 2017] не затрагивает проблему звукового ландшафта города непосредственно. Тем не менее тема звука в городе в ней фигурирует; кроме того, пред- 
ставленный анализ дает возможность через звук и запись звука взглянуть на взаимоотношения гражданина и государства - тема, для городских исследований не новая. В некоторых городах США внедрена система моментальной звуковой фиксации выстрелов. Она позволяет не только отслеживать уровень преступности, но и контролировать, насколько быстро полиция реагирует на происшествия. В 2007 г. в Вашингтоне полицейский, не находящийся на службе, застрелил чернокожего подростка, пытавшегося угнать его мопед. Во время погони полицейскому показалось, что преступник вооружен и собирается стрелять по догоняющим. Данные системы фиксации выстрелов легли в основу расследования и помогли оправдать полицейского. Однако в отчете компании-производителя CCTV-системы упомянуто, что архитектурные сооружения, расположенные на аллее, где был убит подросток, помешали датчикам, и полученные для анализа данные были записаны с помехами. Поэтому есть стороны, полагающие, что полицейский применил оружие необоснованно, и описанный случай есть проявление структурного расизма. Описанный кейс автор рассматривает в терминах паноптикума, в котором у государства есть монопольное право на насилие, и роль городского пространства в этом процессе.

Этот кейс демонстрирует, что на работу таких систем, как фиксация выстрелов, надо смотреть не только и не столько через призму приватности/прозрачности. Автор приходит к выводу, что такие системы используют и трансформируют логику и практики управления и господства, через которые часто рассматриваются, например, CCTV-камеры в городе. В работе приводятся ссылки на исследования, которые показывают, что внедрение систем фиксации выстрелов влечет за собой рост агрессии со стороны полицейских. Остается не вполне ясным, как именно собираются и анализируются эти данные, но очевидно, что они могут быть использованы ксенофобами и расистами для оправдания агрессии со стороны представителей власти, как в случае с убийством чернокожего подростка в $\mathrm{Ba}$ шингтоне.

Немалая часть эмпирических работ о звуке в городе посвящена изучению восприятия горожанами окружающего звукового ландшафта (далее мы приводим описание нескольких количественных исследований на эту тему). Работа “A grounded theory approach to the subjective understanding of urban soundscape in Sheffield" [Liu, Kang, 2016] примечательна с точки зрения метода, который выбран авторами - это так называемая обоснованная теория (grounded theory approach). В отличие от большей части качественных методов этот подход предлагает вывести теоретические заключения на основе эмпирических данных, иначе говоря, является индуктивным. Ключевой вывод исследования, который позволяет получить выбранная методика, заключается в том, что, оценивая звук, индивиды зачастую опираются не на его физические характеристики, а на собственный положительный или негативный опыт, с ним связанный.

На примере нескольких работ мы уже выяснили, как звук может стать причиной конфликта в городском сообществе. В качестве предыстории к статье "Parenting and neighbouring in the consolidating city: The emotional geographies of sound in apartments" [Kerr, Gibson, Klocker, 2018] приводится случай, когда причиной конфликта - на этот раз между жителями одного дома - стал детский крик. Высокая плотность населения является одним из неотъемлемых атрибутов города; это, в свою очередь, влияет на стоимость земли и стоимость недвижимости. В результате домохозяйства все чаще склонны отказываться от отдельного дома в пользу апартаментов в многоквартирных домах. Авторы этой статьи выбрали предметом своего исследования эмоциональный опыт членов семей, живущих в апартаментах с детьми. В ходе исследования в глубинном интервью поучаствовали 17 домохозяйств, проживающих в Сиднее и его пригородах: исследователей интересовали мотивы выбора именно этого вида жилья, а также опыт, который переживают люди, вынужденные делить жилое пространство как с близкими, так и с соседями по дому. Проблемы, связанные с шумом, стали центральными в ответах информантов. Желая оставаться одновременно хорошими родителями и хорошими соседями, все они так или иначе выработали ряд практик по «управлению звуком» в своей квартире в любое время суток. Их можно условно разделить на две категории. Прежде всего, это выработанные в каждой семье практики жизни с ребенком. Так, одна из молодых мам отметила, что они с мужем научились максимально быстро реагировать на ночной плач сына. Другие респонденты ограничивали время шумных игр ребенка. Вторая категория таких практик связана 
с преобразованием пространства. Пары обращали внимание на материал стен при выборе квартиры, а после рождения ребенка старались максимально изолировать детский шум в помещении, покрывая пол ковром, затворяя окна или отводя для детских занятий комнату, не граничащую с другими квартирами.

Эффективность жилищной политики, как правило, оценивается с помощью формальных количественных показателей (доступность жилья, количество жилой площади в расчете на единицу численности населения и т.д.). Работы, подобные этой, отражают мнение отдельных владельцев или нанимателей жилья; в данном случае такой подход позволяет выявить отдельные недостатки жилищной политики в рамках города и трансформации практик горожан, к которым эти недостатки приводят.

Количественные исследования городского звука, в отличие от всех описанных выше работ, носят сугубо прикладной характер и по своей тематике ближе скорее к urban planning, чем к urban studies. Поэтому нам показалось важным представить их в отдельном разделе обзора.

В статье "Green and calm: Modeling the relationships between noise pollution propagation and spatial patterns of urban structures and greencovers" [Sakieh et al., 2017] описана попытка смоделировать зависимость между уровнем шумового загрязнения и количественными характеристиками городской среды. Данные для исследования собирались в иранском городе Кередж. По ряду причин в течение последних десятилетий город пережил стремительный рост населения (по словам авторов, в среднем до 3,1\% в год). Это обусловило развитие как экономики города, так и городской среды. Вместе с развитием пришли и стандартные проблемы крупных городов: в частности, продуктом развития транспортной системы стал достаточно высокий уровень шума в городе. В течение одного сезона исследователи замеряли уровень шума в тридцати точках города, а затем с помощью ГИС и стандартных статистических методов - корреляционного и регрессионного анализа - протестировали гипотезу о зависимости этих показателей от плотности и размеров площади застройки и зеленых насаждений, плотности и протяженности улично-дорожной сети, интенсивности транспортных потоков и других измерений. Вывод работы относительно очевиден: связные тер- ритории с высокой плотностью озеленения поблизости от значимых источников шума помогают снизить их воздействие. В районах жилой застройки уровень шума в среднем ниже в кварталах со сравнительно низкой плотностью застройки и зелеными зонами.

Зеленые насаждения не только снижают воздействие внешнего шумового загрязнения - об этом пишут [Aletta et al., 2016] в своей работе "Differences in soundscape appreciation of walking sounds from different footpath materials in urban parks”. C 2002 г. в городах стран Евросоюза ведется политика выявления и сохранения «тихих зон». Зачастую таковыми становятся парки. Таким образом, у городских управленцев в сфере благоустройства возникает задача не только измерять уровень шума в парках, но и управлять им. Одним из источников звука в этих локациях выступает покрытие пешеходных дорожек. В рамках лабораторного эксперимента 88 респондентов протестировали четыре наиболее популярных материала для устройства парковых дорожек: дерево, гравий, камень и траву. Отдельный интерес представляет дизайн эксперимента: в течение единицы времени респонденты слушали музыку через наушники, в то время как вокруг них постоянно прогуливались 12 человек. Затем информантов просили оценить, насколько их раздражала окружающая звуковая среда. Во второй части эксперимента сами респонденты во время пешей прогулки оценивали акустический фон, создаваемый разными типами покрытий. Таким образом, исследование позволило сравнить, насколько по-разному участники воспринимают один и тот же звук, когда его издают окружающие и когда они производят его сами. Выяснилось, что восприятие в этих двух ситуациях действительно отличается: существенно меньше информантов раздражал звук, издаваемый ими самими во время прогулки. Что касается непосредственно материалов, то выше всего пользователи оценили травяное покрытие дорожек, наиболее неудовлетворительное же качество звука, по их мнению, создает гравий.

Проблему перехода от измерения уровня шума к управлению им продолжает работа "Soundscape assessment of a monumental place: A methodology based on the perception of dominant sounds" [Pérez-Martínez, Torija, Ruiz, 2018]. Цель исследования состояла в том, чтобы выяснить, как пользователи определенных городских пространств реагируют 
на звуки среды в зависимости от того, что является их источником. Для изучения был выбран комплекс исторических памятников на о. Гренада. В опросе о восприятии окружающей звуковой среды поучаствовали 400 респондентов, при этом было выбрано двадцать точек для проведения опросов, различных с точки зрения интенсивности дорожного движения и пешеходных потоков, наличия источников воды и птиц. Результат исследования совпадает с ожиданиями: субъективно наиболее приятными посетители называли звуки природы, тогда как более всего их раздражал шум толпы. В случаях, когда более 50\% опрошенных идентифицировали звук как антропогенный, общая удовлетворенность звуковым ландшафтом значительно снижалась.

В городской среде в целом значимых источников шума куда больше, нежели в парке или в пределах туристического центра. Однако едва ли архитекторы учитывают это при проектировании общественных пространств. Статья "On the ability to correlate perceived sound to urban space geometries" [Calleri et al., 2016], как и многие, описанные здесь, вновь обращается к специфике восприятия городской среды. Отличие ее состоит в том, что предметом исследования здесь выступает не звук, а его «контейнер» - городское пространство, а именно геометрия пространства. Цель работы заключалась в том, чтобы понять, воспринимают ли участники эксперимента окружающую городскую среду аудиально, не имея возможности ее увидеть. Если да, то насколько детальным является это восприятие и какие факторы на него влияют? Участникам интервью были предложены аудиозаписи звукового ландшафта трех городских пространств: переулка, широкой улицы и городской площади. Во всех случаях звуковые фрагменты записывались ночью, чтобы сохранить возможность изучить только геометрию пространства, вне зависимости от ее наполнения. В рамках эксперимента было необходимо сопоставить услышанные звуки с пространствами, в пределах которых они могли быть записаны. Затем был проведен базовый статистический анализ полученных ответов. Обобщая результаты, исследователи утверждают, что статистически значимая разница в восприятии звука обнаруживается только в сравнении улицы и площади. В остальных случаях респонденты, как правило, не могли сопоставить изменение звука с изменением пространства. Однако стоит сказать, что авторы несколько более позднего исследования - “Using Virtual Reality for assessing the role of noise in the audio-visual design of an urban public space" [Sanchez et al., $2017]$ - более уверенно говорят о влиянии звуковой составляющей на общее восприятие среды. Дизайн этого исследования в целом схож с предыдущей работой, с той лишь разницей, что в последнем эксперименте была использована технология виртуальной реальности. Она позволяла оценивать восприятие изменений как визуальной, так и звуковой среды города. Итоги работы показывают, что жители замечают изменение звука, в частности уровня шума, однако доминирующим фактором при определении общей оценки изменений все же является визуальная сторона: привлекают внимание появление/изменение объектов и зеленые насаждения.

Пример исследований звука в городе и научного журнала “Sound Studies” опровергает наше первоначальное предположение о том, что появление академического издания, посвященного некоторой исследовательской области, априори становится драйвером ее развития. В данном случае, напротив, журнал не отражает развитие профильной области знания: наиболее яркие, на наш взгляд, исследования звукового ландшафта городов за весь рассматриваемый период появлялись в журналах, не связанных с изучением звука. Тем не менее эти работы дают читателю возможность по-новому взглянуть на весь предшествующий опыт исследования и проектирования городов. В нашем обзоре мы постарались показать, как в контексте звука по-новому раскрываются, казалось бы, уже привычные проблемы и концепты urban studies, такие как право на город, социально-пространственная дифференциация населения, общественный выбор и т.д. Авторы используют для ответа на поставленные исследовательские вопросы методы интервью, анализ кейсов, картографирование, статистический анализ. Не менее важной тематика звука оказывается и в публикациях по городскому планированию, и здесь исследователи при помощи примерно того же набора инструментов отвечают на вопросы об оптимальной, с точки зрения восприятия звука, планировке пространств или таком феномене, как управление звуковой средой в городе. Так или иначе, проанализированные работы презентуют отдельные кейсы или готовые подходы планирования и управления городами, приводящие к росту 
благосостояния горожан. Уже одно только это легитимирует и обосновывает необходи- мость существования такой научной области, как исследования звука в городе.

\section{Источники}

Вебер М. (2001) История хозяйства. М.: КАНОН-ПРЕСС. Вирт Л. (2005) Урбанизм как образ жизни // Вирт Л. Избранные работы по социологии. М.; СПб. С. 93118.

Aletta F., Kang J., Astolfi A., Fuda S. (2016) Differences in soundscape appreciation of walking sounds from different footpath materials in urban parks // Sustainable Cities and Society. No. 27. P. 367-376.

Bullen R., Fricke F. (1976) Sound propagation in a street // Journal of Sound and Vibration. Vol. 46 (1).P. 33-42.

Calleri C.,Astolfi A., Armando A., Shtrepi, L. (2016) On the ability to correlate perceived sound to urban space geometries // Sustainable Cities and Society. No. 27. P. 346-355.

Dreckmann K. (2017) The pop cultural materialization of sound in the museum: Exhibition "Rock und Pop im Pott", Essen/Germany, Ruhr Museum // Sound Studies. Vol. 3 (1). P. 88-90.

Foucalt M. (1970) The Order of Things. N. Y.: Vintage Books / Random House.

Gerloff F. (2016) Adventures in sonic publishing: Provoke! Digital Sound Studies / Whitney Anne Trettien, Darren Mueller, Mary Caton Lingold (eds). Sound Studies. Vol. 2 (1). P. 101-103.

Galloway K. (2015) Materiality and aural memory in the Harbour Symphony (St. John's, Newfoundland) // Sound Studies. Vol. 1 (1). P. 118-143.

Harvey D. (2003) The right to the city // International Journal of Urban and Regional Research. Vol. 27 (4). P. 939-941.

Kerr S.M., Gibson C., Klocker N. (2018) Parenting and neighbouring in the consolidating city: The emotional geographies of sound in apartments // Emotion, Space and Society. No. 26. P. 1-8.

Liu F., Kang J. (2016) A grounded theory approach to the subjective understanding of urban soundscape in Sheffield // Cities. No. 50. P. 28-39.

Madrid A.L. (2016) Landscapes and gimmicks from the "sounded city": listening for the nation at the sound archive // Sound Studies. Vol. 2 (2). P. 119-136.

Merrill A. (2017) The Life of a Gunshot: Space, Sound and the Political Contours of Acoustic Gunshot Detection // Surveillance \& Society. Vol. 15 (1). P. 42.
Morat D. (2016) The Roaring Twenties online: The Roaring Twenties. An interactive exploration of the his torical soundscape of New York City / E. Thompson, designed by Scott Mahoy // Sound Studies. Vol. 2 (1). P. $98-100$.

Mumford L. (1938) The Culture of cities.

Nyerges A. (2016) Hearing Hart Crane: in the shape of New York's noise // Sound Studies. Vol. 2 (2). P. 107-118.

Patsarika M., Schneider T., Edwards M. (2018) 'If I was King of India I would Get All the Horns Out of Cars': A Qualitative Study of Sound in Delhi // International Journal of Urban and Regional Research. Vol. 42 (1). P. 74-89.

Perevedentseva M., Garcia L.M. (2016) Chicago house music, from dance floor to museum: the Frankie Knuckles Vinyl Collection: The Frankie Knuckles Vinyl Collection at the Stony Island Arts Bank, 6760 South Stony Island Avenue, Chicago // Sound Studies. Vol. 2 (1). P. $95-98$.

Pérez-Martínez G., Torija A.J., Ruiz D.P. (2018) Soundscape assessment of a monumental place: A methodology based on the perception of dominant sounds // Landscape and Urban Planning. No. 169. P. 12-21.

Perkins A. (2015) Muslim sound, public space, and citizenship agendas in an American City. Citizenship Studies. Vol. 19 (2). P. 169-183.

Sakieh Y., Jaafari S., Ahmadi M., Danekar A. (2017) Green and calm: Modeling the relationships between noise pollution propagation and spatial patterns of urban structures and green covers. Urban Forestry \& Urban Greening. No. 24. P. 195-211.

Sanchez G.M.E., Van Renterghem T., Sun K., De Coensel B., Botteldooren D. (2017) Using Virtual Reality for assessing the role of noise in the audio-visual design of an urban public space // Landscape and Urban Planning. No. 167. P. 98-107.

Murray Schafer R. (1977) The soundscape: Our sonic environment and the tuning of the world. Vancouver: Destiny Books.

Thompson E.A. (2004) The soundscape of modernity: architectural acoustics and the culture of listening in America, 1900-1933. MIT Press. 


\title{
NIKITA KOSOLAPOV \\ THE CUTTING-EDGE: A REVIEW OF RECENT ACADEMIC PAPERS ON URBAN SOUND STUDIES
}

\author{
Nikita Kosolapov, Master's student, Vysokovsky Graduate School of Urbanism, HSE; 13 bldg. 4 Myasnitskaya Street, \\ Moscow, 101000, Russian Federation. \\ E-mail:nakosolapov@gmail.com \\ Abstract \\ This article describes a number of research papers in the field of urban sound studies 2015-2018. \\ The interdisciplinary journal Sound Studies was taken as a point of departure, however, papers from several other \\ journals are also discussed. The manner of posing questions and research approaches are the main focus \\ of the paper.
}

Key words: sound studies; urban studies; interdisciplinary research; academic journals

\section{References}

Aletta F., Kang J., Astolfi A., Fuda S. (2016) Differences in soundscape appreciation of walking sounds from different footpath materials in urban parks. Sustainable Cities and Society, no 27, pp. 367-376.

Bullen R., Fricke F. (1976) Sound propagation in a street. Journal of Sound and Vibration, vol. 46 (1), pp. 33-42.

Calleri C., Astolfi A., Armando A., Shtrepi L. (2016) On the ability to correlate perceived sound to urban space geometries. Sustainable Cities and Society, no 27, pp. 346-355.

Dreckmann K. (2017) The pop cultural materialization of sound in the museum: Exhibition "Rock und Pop im Pott”, Essen/Germany, Ruhr Museum. Sound Studies, vol. 3 (1), pp. 88-90.

Foucalt M. (1970) The Order of Things. New York:Vintage Books / Random House.

Gerloff F. (2016) Adventures in sonic publishing: Provoke! Digital Sound Studies / Whitney Anne Trettien, Darren Mueller, Mary Caton Lingold (eds). Sound Studies, vol. 2 (1), pp. 101-103.

Galloway K. (2015) Materiality and aural memory in the Harbour Symphony (St. John's, Newfoundland). Sound Studies, vol. 1 (1), pp. 118-143.

Harvey D. (2003) The right to the city. International Journal of Urban and Regional Research, vol. 27 (4), pp. 939-941.

Kerr S.M., Gibson C., Klocker N. (2018) Parenting and neighbouring in the consolidating city: The emotional geographies of sound in apartments. Emotion, Space and Society, no 26, pp. 1-8.
Liu F., Kang J. (2016) A grounded theory approach to the subjective understanding of urban soundscape in Sheffield. Cities, no 50, pp. 28-39.

Madrid A.L. (2016) Landscapes and gimmicks from the "sounded city": listening for the nation at the sound archive. Sound Studies, no 2 (2), pp. 119-136.

Merrill A. (2017) The Life of a Gunshot: Space, Sound and the Political Contours of Acoustic Gunshot Detection. Surveillance \& Society, vol. 15 (1), p. 42.

Morat D. (2016) The Roaring Twenties online: The Roaring Twenties. An interactive exploration of the historical soundscape of New York City / E. Thompson, S. Mahoy (designer). Sound Studies, vol. 2 (1), pp. 98-100.

Mumford L. (1938) The Culture of cities.

Nyerges A. (2016) Hearing Hart Crane: in the shape of New York's noise. Sound Studies, vol. 2 (2), pp. 107118.

Patsarika M., Schneider T., Edwards M. (2018) 'If I was King of India I would Get All the Horns Out of Cars': A Qualitative Study of Sound in Delhi. International Journal of Urban and Regional Research, vol. 42 (1), pp. 74-89.

Perevedentseva M., Garcia L.M. (2016) Chicago house music, from dance floor to museum: the Frankie Knuckles Vinyl Collection: The Frankie Knuckles Vinyl Collection at the Stony Island Arts Bank, 6760 South Stony Island Avenue, Chicago. Sound Studies, vol. 2 (1), pp. 95-98.

Pérez-Martínez G., Torija A.J., Ruiz D.P. (2018) Soundscape assessment of a monumental place: A methodology 
based on the perception of dominant sounds. Landscape and Urban Planning, no 169, pp. 12-21.

Perkins A. (2015) Muslim sound, public space, and citizenship agendas in an American City. Citizenship Studies, vol. 19 (2), pp. 169-183.

Sakieh Y., Jaafari S., Ahmadi M., Danekar A. (2017) Green and calm: Modeling the relationships between noise pollution propagation and spatial patterns of urban structures and green covers. Urban Forestry \& Urban Greening, no 24, pp. 195-211.

Sanchez G.M.E., Van Renterghem T., Sun K., De Coensel B., Botteldooren D. (2017) Using Virtual Reality for assessing the role of noise in the audio-visual design of an urban public space. Landscape and Urban Planning, no 167, pp. 98-107.

Murray Schafer R. (1977) The soundscape: Our sonic environment and the tuning of the world. Vancouver: Destiny Books.

Thompson E.A. (2004) The soundscape of modernity: architectural acoustics and the culture of listening in America, 1900-1933. MIT Press.

Weber M. (2001) History of economy. Moscow: KanonPress.

Wirth L. (2005) Urbanism as a way of life. Selected texts of sociology, pp. 93-118. 